\title{
Objetivos de la UNESCO y la Unión Europea sobre Gender Mainstreaming. Aplicación en la docencia del Derecho mercantil
}

\author{
María Isabel Huerta Viesca
}

Universidad de Oviedo, España, ishuerta@uniovi.es

\begin{abstract}
Resumen
La Unión Europea y la UNESCO tienen como objetivo principal el desarrollo de políticas educativas igualitarias que propicien, a su vez, una sociedad más justa e igualitaria. Para ello se insiste incansablemente, en especial en estos últimos años, en la necesidad de desarrollar políticas educativas y formativas que incluyan, en todas las disciplinas, el Gender Mainstreaming. Para las materias jurídicas, y en especial para el Derecho mercantil y del comercio, es esencial contar con obras didácticas y programas que incorporen esta perspectiva transversal. A su análisis y propuesta se dirige este trabajo.
\end{abstract}

Palabras clave: Gender Mainstreaming, Derecho de la empresa, Derecho mercantil, docencia, igualdad, sociedad justa e igualitaria.

\section{Introducción}

Se propone una reflexión sobre la necesidad de llevar a la práctica fórmulas educativas que incorporen la perspectiva transversal de género e igualdad en todas las materias y disciplinas. Esa propuesta puede ser especialmente valiosa en la docencia relacionada con la empresa y el mundo de los negocios, también en la Universidad. En España se carece de obras específicas que contribuyan a impedir el sesgo de género que se produce en este campo en la realidad práctica. La constitucionalización de la igualdad de género, legal y formal, se muestra insuficiente para superar la brecha real de género existente en el ámbito empresarial social. Se valorará la importancia esencial de las fórmulas educativas de Gender Mainstreaming y su repercusión en esta materia. 


\section{Derecho a la educación y desarrollo jurídico de políticas educativas igualitarias}

La segregación de género en el ámbito laboral y empresarial persiste y es una constante, aunque en grados muy distintos, muchas veces con formas sutiles, en todo el mundo. Concretamente, en el campo empresarial esta segregación se observa en varios apartados. Por un lado, se constata la menor actividad emprendedora femenina en la praxis empresarial, lo que supone -desde el punto de vista económico- un freno para el desarrollo económico de las personas, las familias, las sociedades y los Estados. Por otro lado, el emprendimiento femenino no tiene el mismo desarrollo en comparación con el masculino. Ello no ocurre siempre por falta de formación empresarial, pues en la actualidad la formación femenina alcanza cotas históricas igualitarias en los Estados occidentales (no es así en otras zonas del planeta, en que la paridad educativa no existe ${ }^{1}$ ), y también en el caso español. Lo que se produce es más bien un sesgo empresarial de género por motivos tanto educacionales -las mujeres se dirigen hacia actividades más concretas y limitadas que los hombres-, como sociales y económicos estructurales -a los que contribuye también la fuerza de la regulación jurídica mercantil- que impiden que las mujeres puedan acceder en la práctica a los puestos de mando o de poder de las organizaciones empresariales.

Ha de fomentarse la cultura emprendedora femenina en beneficio individual y social. Para ello, como requisito esencial previo, es absolutamente imprescindible un cambio formativo de hombres y mujeres en lo que respecta al mundo de la empresa. Sin embargo, y a pesar de la igualdad formal entre todas las personas que establecen los sistemas jurídicos occidentales, persisten, incluso en los más avanzados social y económicamente, altas -y en ocasiones, casi insuperables- barreras de género enraizadas en estereotipos erróneos que se perpetúan en el tiempo. Como ha expresado la Unión Europea en su Recomendación general 36 (2017) sobre el derecho de las niñas y las mujeres a la educación, "la dimensión de la igualdad reviste particular importancia porque la sociedad forja las desigualdades de género y las reproduce a través de las instituciones sociales, muy en particular las educativas. En muchas sociedades, en lugar de cuestionar las arraigadas normas y prácticas que discriminan por razón de género, la escolarización refuerza los estereotipos sobre los hombres y las mujeres y preserva el orden de género de la sociedad reproduciendo las jerarquías femenino/masculino y subordinación/dominación y las dicotomías reproducción/producción y privado/público".

1 El 8 de marzo de 2018 UNESCO present su Informe de segumiento de la educación en el mundo, http://www.rightto-education.org/es/news/presentaci-n-del-resumen-sobre-g-nero-2018-de-la-unesco, en el que se comprueba la lentitud del progreso hacia la igualdad de género en la educación, en la que solo el $44 \%$ de los países se ha comprmetido plenamente con la pariddad de género. Vid. UNESCO eAtlas of Literacy, Unesco Institute for Statistics, https://tellmaps.com/uis/literacy/\#!/tellmap/-601865091 De los 758 millones de adultos analfabetos, dos tercios siguen siendo mujeres. 
El derecho a la educación de las mujeres está reconocido en numerosos textos internacionales que, una vez aprobados por los Estados, como es el caso de España, pasan a formar parte de los Ordenamientos Jurídicos internos a un nivel máximo en la jerarquía normativa. Ello significa que serán aplicables directamente y además servirán como criterio axiológico interpretativo de las normas internas estatales. Entre esos textos hay que citar varios muy relevantes. En el seno de la ONU, la UNESCO, Organización de las Naciones Unidas para la Educación, la Ciencia y la Cultura, mantiene los mismos altos objetivos con respecto a la materia de género e igualdad de las personas. Desde un punto de vista general, el Pacto Internacional de Derechos Económicos, Sociales y Culturales de $1966^{2}$ reconoce en su artículo 13 el derecho de toda persona a la educación, que debe orientarse hacia el pleno desarrollo de la personalidad humana y del sentido de su dignidad y debe fortalecer el respeto por los derechos humanos y las libertades fundamentales. Por su parte, la Carta de los Derechos Fundamentales de la Unión Europea (2000), que se convirtió en norma jurídica vinculante en la Unión Europea con la entrada en vigor del Tratado de Lisboa, en diciembre de 2009, por lo que tiene el rango jurídico de los Tratados de la Unión Europea, establece en su artículo 23 que la igualdad entre hombres y mujeres debe ser garantizada en todos los ámbitos, y que "el principio de igualdad no impide el mantenimiento o la adopción de medidas que ofrezcan ventajas concretas en favor del sexo menos representado". Las normas generales expresadas atienden implícita e indirectamente a la necesidad de superar sesgos en la educación de modo que promueva la dignidad de la persona y el desarrollo de sus derechos y libertades fundamentales.

Desde una perspectiva más concreta referida a los métodos y programas docentes para supercar estereotipos y sesgos en la educación es esencial la Convención sobre la Eliminación de Todas las Formas de Discriminación contra la Mujer (Nueva York, 18 de diciembre de 1979) ${ }^{3}$. Este es el único Tratado jurídicamente vinculante a nivel internacional que se centra exclusivamente en los derechos de la mujer. Establece que los Estados Partes tomarán todas las medidas apropiadas para eliminar la discriminación contra la mujer ${ }^{4}$, a fin de asegurarle la igualdad de derechos con el hombre en la esfera de la educación y en particular para asegurar, en condiciones de igualdad entre hombres y mujeres la eliminación de todo concepto estereotipado de los papeles masculino y femenino en todos los niveles y en todas las formas de enseñanza, mediante el estímulo de la educación mixta y de otros tipos de educación que

2 Instrumento de ratificación por España publicado en el Boletín Oficial del Estado de 30 de abril de 1977. Cabe citar también la Convención sobre los Derechos de las Personas con Discapacidad, de 2006, que en su artículo 6 obliga a los Estados Partes a adoptar medidas para asegurar que todas las mujeres con discapacidad puedan disfrutar plenamente y en igualdad de condiciones de todos los derechos humanos y libertades fundamentales, y tomar "todas las medidas pertinenetes para asegurar el pleno desarrollo, adelanto y potenciación de la mujer, con el propósito de garantizarle el ejercicio y goce de los derechos humanos y las libertades fundamentales"; la Convención Europea de Derechos Humanos (1950); o el Convenio Europeo de Derechos Humanos (1958).

3 Instrumento de ratificación por España de 16 de diciembre de 1983 publicado en el Boletín Oficial del Estado de 16 de diciembre de 1983.

4 RTE, Iniciativa por el Derecho a la Educación (2018). http://www.right-to-education.org/es/issuepage/marginalised-groups/las-ni-y-las-mujeres 
contribuyan a lograr este objetivo. Esta Convención de Naciones Unidas sobre la Eliminación de todas las formas de Discriminación contra la Mujer ${ }^{5}$ declara en sus primeras palabras que "la máxima participación de la mujer, en igualdad de condiciones con el hombre, en todos los campos, es indispensable para el desarrollo pleno y completo de un país, el bienestar del mundo y la causa de la paz". Los Estados Partes están obligados a coadyuvar a la modificación de los patrones socioculturales de conducta de hombres y mujeres para eliminar "los prejuicios y las prácticas consuetudinarias y de cualquier otra índole que estén basados en la idea de la inferioridad o superioridad de cualquiera de los sexos o en funciones estereotipadas de hombres y mujeres" (artículo 5), y deberán tomar medidas necesarias para eliminar en su Derecho interno y en sus estructuras sociales los estereotipos de género": "los Estados Partes tomarán todas las medidas apropiadas para: a) modificar los patrones socioculturales de conducta de hombres y mujeres, con miras a alcanzar la eliminación de los prejuicios y las prácticas consuetudinarias y de cualquier otra índole que estén basados en la idea de la inferioridad o superioridad de cualquiera de los sexos o en funciones estereotipadas de hombres y mujeres". Todas las disposiciones de la Convención que afirman la igualdad de responsabilidades de ambos sexos en la vida familiar e iguales derechos con respecto a la educación y al empleo, atacan enérgicamente los patrones culturales que definen el ámbito público como un mundo masculino y la esfera doméstica como el dominio de la mujer. Su artículado obliga a la modificación de los libros, programas escolares y métodos de enseñanza para eliminar los conceptos estereotipados en la esfera de la educación.

El artículo 10, Parte III, de la Convención, dice: "Los Estados Partes adoptarán todas las medidas apropiadas para eliminar la discriminación contra la mujer, a fin de asegurarle la igualdad de derechos con el hombre en la esfera de la educación y en particular para asegurar, en condiciones de igualdad entre hombres y mujeres: a) Las mismas condiciones de orientación en materia de carreras y capacitación profesional, acceso a los estudios y obtención de diplomas en las instituciones de enseñanza de todas las categorías, tanto en zonas rurales como urbanas; esta igualdad deberá asegurarse en la enseñanza preescolar, general, técnica y profesional, incluida la educación técnica superior, así como en todos los tipos de capacitación profesional". La supresión de los estereotipos de género en la educación y en el desarrollo profesional y el empleo son factores clave en este texto, obligatorios para los Estados Parte y decisivos también a la hora de llevar a cabo la interpretación de las normas jurídicas de cada Ordenamiento. No deben perderse de vista en su aplicación práctica, ni deben quedar relegados

\footnotetext{
5 http://www.un.org/womenwatch/daw/cedaw/text/sconvention.htm
}

6 La Recomendación General 36 (2017) sobre el derecho de las niñas y las mujeres a la educación es muy reveladora: "Sin embargo, las niñas y las mujeres tienen dificultades desmedidas para reivindicar y ejercer su derecho humano fundamental a la educación por diversos factores, entre los que destacan las barreras de acceso que enfrentan las niñas y las mujeres de grupos desfavorecidos y marginados, exacerbadas por la pobreza y las crisis económicas; los estereotipos de género en los planes de estudios, los libros de texto y los procesos pedagógicos; la violencia contra las niñas y las mujeres dentro y fuera de la escuela; y los obstáculos de orden estructural e ideológico para que se decanten por disciplinas académicas o de formación profesional dominadas por los hombres". 
a una declaración de principios simplemente voluntariosa o de escaparate. Por el contrario, debe insistirse en su valor aplicativo real y en la obligación que implica para los Estados parte, como España. Por otro lado, las instituciones, también la Universidad, y los organismos públicos y privados han de tender al respeto y a la consecución de los objetivos plasmados en estas normas internacionales. De ahí que los estudios y las investigaciones sobre género en sus implicaciones en la empresa, el empleo, la publicidad y los demás ámbitos jurídicos mercantiles y el acceso a los puestos de dirección de las empresas y organizaciones sean factores esenciales en el acceso a la igualdad real -y no sólo formal- de las personas. Y de ahí también que la esfera docente, metodológica y discente deba incorporar, en todas las etapas educativas y formativas, la perspectiva del gender mainstreaming con el fin de lograr la plena igualdad en beneficio social y, sobre todo, para la realización de la dignidad de todas las personas.

\section{Educación e igualdad de género en la doctrina y en la docencia del Derecho Mercantil y de la Empresa en España}

En el ámbito del Derecho mercantil la perspectiva de género casi no se ha abordado en España, ni desde el punto de vista de la investigación ni desde la perspectiva docente, hasta los últimos años de finales del siglo XX y principios del XXI. Anteriormente, los estudios doctrinales, que se fueron desgranando incesantemente a lo largo de los años con las sucesivas reformas legislativas y cambios axiológicos y normativos, apenas prestaron atención a las cuestiones de diversidad o de género, y menos aún en el ámbito de las materias jurídicas mercantiles o de la empresa.

La Constitución de 1931 recogió diversos preceptos legislativos que igualaban a todos los españoles ante la ley -artículo 2- y que prohibían privilegios jurídicos por la naturaleza, la filiación, el sexo, la clase social, la riqueza, las ideas políticas ni las creencias religiosas artículo 25-; además declaró constitucionalmente la igualdad de derechos de ambos sexos en el matrimonio -artículo 43-. Fue especialmente relevante el artículo 40 de aquella Constitución, que aseguraba el derecho de ejercicio de una profesión con independencia del género ${ }^{7}$. Estos preceptos dieron lugar a estudios y comentarios doctrinales pero en la realidad el marido seguía siendo el representante legal de la mujer. La brevedad de esta etapa política impidió un desarrollo y una verdadera aplicación de aquellas progesistas -para su tiempo- normas constitucionales.

7 Vid. Ruiz Franco, Rosario (2003). Mujeres y hombres en la España franquista: sociedad, economía, política, cultura, Editorial Complutense, Madrid, 118. 
Durante el regimen franquista se realizaron varios estudios jurídicos al hilo de las viejas normas jurídicas mercantiles que prohibían a la mujer casada el ejercicio del comercio $^{8}$ si no contaba con la autorización marital ${ }^{9}$, casi siempre proponiendo su mantenimiento. Fue necesario esperar hasta los años setenta del siglo XX para que la influencia de las corrientes jurídicas europeas igualizadoras, la fuerza de los movimientos feministas y los cambios sociales -también religiosos y culturales- y económicos en los Estados occidentales de nuestro entorno -importantes receptores de emigración española- y en España, propiciaran el rechazo de la posición de inferioridad de las mujeres en favor de la igualdad de ambos cónyuges en el matrimonio. La Ley de 2 de mayo de 1975 suprimió por fin la necesaria obediencia de la mujer al esposo, además de que a partir de la entrada en vigor de la misma la mujer ya no perderia su nacionalidad por razón del matrimonio ${ }^{10}$.

8 Recordemos que el artículo 4 del Código de Comercio inhabilitaba a las mujeres casadas -no así a viudas o solteraspara ejercer el comercio, estableciéndose en los artículos 6 a 12 los requisitos de la licencia marital para que pudieran ejercitar dichas actividades comerciales.

9 Estudios frecuentemente acogedores de la utilidad de la autorización marital que se consideraba un baluarte para defender la unidad de la familia y el poder de dirección del marido. La Ley de Derechos Políticos, Profesionales y del Trabajo de la Mujer de 1961 permitía que un juez de primera instancia declarara inválida la negativa del marido a dar su autorización marital si hubiera incurrido en abuso de Derecho, si bien la solicitud debía hacerla la esposa contra el marido. La interpretación de la norma fue muy restrictiva y, como es natural, sus resultados prácticos efectivos fueron muy pobres. Cabe destacar la creación, en 1967, de la Asociación Española de Mujeres Juristas, presidida por María Telo Núñez (1915-2014), que insistía en las desigualdades entre los cónyuges y cuya labor fue relevante en los cambios legislativos que se llevarían a cabo posteriormente.

10 Son expresivas las palabras de Telo Núñez, María (1998). "Las investigadoras de la mujer", Diario ABC, 18 de enero de 1998: "Cuando en septiembre de 1969 yo inicie la lucha por liberar a la mujer de las cadenas jurídicas, su situación era tan vejatoria y alienante dentro de la familia, con proyección en lo público, que había artículos como el 1263, que situaba a la mujer casada entre los menores, los locos o dementes y los sordomudos que no sabían leer ni escribir. El artículo 57 obligaba a la mujer a obedecer al marido y la licencia marital era como su sombra para todos los actos de la vida. Sin licencia, prácticamente, la mujer sólo podía hacer testamento... Sin licencia, la mujer no podía trabajar, ni cobrar su salario, ni ejercer el comercio, ni ocupar cargos, ni abrir cuentas corrientes en bancos, ni sacar su pasaporte, ni el carnet de conducir, etc. Si contraia matrimonio con extranjero perdia la nacionalidad y era considerada extrajera -aunque no saliese en su vida de España-; entonces se le extendia carta de residente y perdían eficacia sus estudios, no podía ser funcionaria y necesitaba permiso para trabajar. Sin la licencia no podía aceptar o repudiar herencias, aunque fuesen de sus padres, ni pedir su participación, ni ser albacea; ni defenderse ante los tribunales (salvo en juicio criminal) ni defender sus bienes propios, ni vender o hipotecar estos bienes; ni disponer de las gananciales más que para hacer la compra diaria, aunque los gananciales procediesen de su sueldo o salario. Por el contrario, el marido podía disponer libremente de los gananciales (salvo inmuebles o establecimientos mercantiles) y ella no tenía más derecho que a recibir la mitad de lo que quedase al fallecer él. Estaba obligada a seguir al marido dondequiera que él fijase la residencia. No tenía patria potestad sobre los hijos hasta que muriese el padre, e incluso, hasta el año 1970, él podía darlos en adopción sin consentimiento de la madre. Y parece que esto nunca existió o que se hizo solo. No, no se hizo solo. La reforma está contenida en leyes, fuimos muchas las mujeres juristas que trabajamos años hasta la extenuación, sin ayudas ni subvenciones. Primero unas pocas desde una Comisión de Estudios Juridicos y luego desde la Asociación Española de Mujeres Juristas, ambas fundadas y presididas por mi. Divuigábamos el Derecho y conseguimos que por primera vez, la mujer entrase en la Comisión de Codificación del Ministerio de Justicia para estudiar la reforma. Cuando se aprobó la Constitución, que eliminó toda discriminación por razón de sexo, ya existía la Ley del 2 de mayo de 1975, que quitó la obediencia al marido (para lo cual sudé tinta en la Comisión de Codificación), la licencia marital, que convirtio a la mujer en persona: dejó ésta de perder la nacionalidad por razón de matrimonio y barrió casi todas las discriminaciones antes mencionadas, dejando el camino libre para atacar dos fundamentales: la administración de los gananciales y la patria potestad, que juntamente con la filiación, el matrimonio y el divorcio, fueron objeto de las leyes del 13 de mayo de 1981 y 7 de julio del mismo año; pero los anteproyectos en que se basan estas dos leyes, los estudiamos en la Comisión de 
La Constitución Española de 1978 instaura como uno de sus valores principales la igualdad de las personas. Ya se manifiesta así inequívocamente en su artículo primero -en el que se declara que España se constituye como Estado social y democrático de Derecho que propugna la igualdad como uno de los valores fundamentales de su Ordenamiento jurídico-, y se establece expresamente en el artículo 14: "Los españoles son iguales ante la ley, sin que pueda prevalecer discriminación alguna por razón de nacimiento, raza, sexo, religión, opinión o cualquier otra condición o circunstancia personal o social". Las formulas genéricas que se utilizan a lo largo de todo el texto constitucional insisten en ese principio de igualdad ${ }^{11}$. También los tratados internacionales suscritos por España incorporan los principios de igualdad y de no discriminación. Es el caso de la Declaración Universal de Derechos Humanos de 10 de diciembre de 1948 (artículos 1, 2 y 7); el Pacto Internacional de Derechos Civiles y Políticos de 19 de diciembre de 1966 (artículos $2.1^{\circ}$ y 20 20.2, 26 y 27); el Pacto Internacional de Derechos Económicos, Sociales y Culturales de 19 de diciembre de 1966 (artículos $2.2^{\circ}$ y $3^{\circ}$ ); la Convención de las Naciones Unidas sobre los Derechos del Niño de 20 de noviembre de 1959 (artículos 1 y 10); el Convenio Europeo para la Protección de los Derechos Humanos y de las Libertades Públicas de 4 de octubre de 1950 (artículo 17), y la Carta de los derechos fundamentales de la Unión Europea proclamada solemnemente en Niza el 7 de diciembre de 2000 (artículos 20, 21 y 23), junto con los artículos 2 y 3 del Tratado de la Unión Europea ${ }^{12}$.

A partir de estos textos, otros complementarios, y principalmente del artículo 14 de la Constitución Española y también de la Ley Orgánica de Igualdad de 2007, ha recaído una abundante jurisprudencia del Tribunal Constitucional que ha combinado el precepto constitucional con lo que dispone el artículo 9.2 del mismo texto -“corresponde a los poderes públicos promover las condiciones para que la libertad y la igualdad del individuo y de los grupos en que se integra sean reales y efectivas; remover los obstáculos que impidan o dificulten su plenitud y facilitar la participación de todos los ciudadanos en la vida política, económica, cultural y social"-, de forma que en el Ordenamiento Jurídico español se admite la validez constitucional de las medidas de acción positiva y de discriminación inversa frente a colectivos desfavorecidos. Se trata entonces de que dichos colectivos, mediante un trato especial más favorable, vean suavizada o compensada su situación de desigualdad sustancial ${ }^{13}$.

Codificación y lo concluimos el año 1978. Con su promulgación alcanzaron plena eficacia los principios de igualdad de la Constitución... Es curioso que haya cosas que siendo noticia durante años, luego se olviden obstinadamente sin saber bien por qué, pero lo que fue, fue".

11 Vid., en este sentido Gálvez Muñoz, Luis (2011). "Sipnosis artículo 14", http://www.congreso.es/consti/constitucion/indice/sinopsis/sinopsis.jsp?art=14\&tipo=2: "todos los españoles...", "los españoles...", "todo español...", "toda persona...", "nadie podrá ser...", etc.

12 Gálvez Muñoz, Luis (2011). "Sipnosis artículo 14”..

13 Sentencia del Tribunal Constitucional 216/1991, de 14 de noviembre (Recurso de amparo número 1844/1988, Ponente Excmo. Sr. D. Carlos de la Vega Benayas). Vid. Rodríguez Coarasa, Cristina (2003). "Sipnnosis artículo 9 de la Constitución Española", http://www.congreso.es/consti/constitucion/indice/sinopsis/sinopsis.jsp?art=9\&tipo=2. 
Objetivos de la UNESCO y la Unión Europea sobre Gender Mainstreaming. Aplicación en la docencia del Derecho mercantil

También a partir de aquéllos textos encontramos interesantísimos estudios de género en su proyección mercantil y de la empresa, ya muy numerosos, en que la doctrina mercantilista aborda con una perspectiva crítica las normas jurídicas cuya declaración de igualdad formal no se corresponde con la realidad social o práctica.

\section{Propuestas educativas para eliminar los estereotipos de género en el ámbito del Derecho de la empresa y mercantil}

Los Tratados y normas internacionales ofrecen un interesante y fructífero catálogo de propuestas para eliminar los estereotipos de género en el ámbito mercantil y empresarial. En la Recomendación general 36 (2017) sobre el derecho de las niñas y las mujeres a la educación se indica que la discriminación que enfrentan las niñas y las mujeres en la educación es tanto ideológica como estructural, y atender a esta realidad es un aspecto de suma importancia para que las mujeres y las niñas puedan disfrutar de sus derechos a la educación, en la educación y mediante la educación, lo que resulta fundamental porque esas prácticas discriminatorias no solo se ejercen en el plano individual, sino que además están codificadas en la legislación, las políticas y los programas y, por tanto, es el propio Estado el que las perpetúa y aplica. Se recalca que el sistema educativo es uno de los ámbitos que se presta a una transformación que, una vez sea llevada a cabo, puede acelerar los cambios positivos en otras esferas. De conformidad con los artículos 5 y 10 c) de la Convención, el Comité recomienda que los Estados partes redoblen los esfuerzos y adopten medidas proactivas para eliminar de la educación los estereotipos de género que perpetúan la discriminación directa e indirecta de las niñas y las mujeres. A tal fin, deben (a) cuestionar y modificar las ideologías y estructuras patriarcales que impiden que las niñas y las mujeres ejerzan plena y libremente sus derechos a la educación, en la educación y mediante la educación, y los disfruten; (b) elaborar y aplicar políticas y programas, incluidas campañas de sensibilización y educativas acerca de la Convención, las relaciones de género y la igualdad de género, en todos los niveles de la enseñanza y en la sociedad en general, dirigidos a modificar los patrones socioculturales de conducta de hombres y mujeres con miras a alcanzar la eliminación de los prejuicios y las prácticas consuetudinarias, en consonancia con lo dispuesto en el artículo 5 a) de la Convención; (d) elaborar planes de estudios, libros de texto y material didáctico que no contengan estereotipos, y revisar los existentes, con objeto de eliminar los estereotipos de género tradicionales que reproducen y refuerzan la discriminación por razón de género de las niñas y las mujeres y de promover una imagen y una voz más equilibrada, exacta, saludable y positiva de las mujeres y las niñas; (e) Implantar, en todos los niveles de la enseñanza, capacitación obligatoria del personal docente sobre las cuestiones de género y la sensibilidad a esas cuestiones y sobre los efectos de las conductas con sesgo de género en los procesos de enseñanza y aprendizaje. 
En particular, incide en la tendencia, que se prolonga en el plano de la participación de las mujeres en los procesos políticos y de adopción de decisiones, debido a que, como las mujeres están insuficientemente representadas, no pueden influir de manera efectiva en las políticas que las afectan: en 2017, la proporción de mujeres en cargos públicos de elección o designación a nivel mundial era de aproximadamente una mujer por cada cuatro hombres. En las cámaras bajas de los Parlamentos las mujeres ocupan el 23,4\% de los escaños, y en las cámaras altas el $22,9 \%$. La representación de las mujeres como consejeras o presidentas en los consejos de administración de las entidades públicas y privadas sigue la misma tendencia. Se continúa marginando en gran medida a las mujeres en la esfera política y en los consejos de administración en razón de leyes, prácticas, actitudes y estereotipos de género discriminatorios.

Para evitarlo, el Comité recomienda que los Estados Parte apliquen, entre otras, las siguientes medidas: a) Formar al personal docente para que adopte estrategias constructivistas de enseñanza que doten a las niñas y las mujeres de capacidad de pensamiento crítico y de un sentimiento positivo de autoestima y confianza para participar en condiciones de igualdad con los hombres en puestos directivos y decisorios en las esferas social, económica y política; b) Adaptar las opciones y los contenidos en la educación de las niñas y las mujeres, en particular en los niveles superiores de la enseñanza, a fin de aumentar su representación en las disciplinas científicas, técnicas y de gestión y, con ello, su calificación, para que puedan acceder a puestos directivos y decisorios, en particular en las profesiones y empleos dominados por los hombres; c) Fortalecer la educación cívica y ciudadana en las escuelas, así como los programas de alfabetización continua de adultos con perspectiva de género destinados a mejorar el papel y la participación de las mujeres en la familia y en la sociedad; d) Reconocer la importancia de empoderar a todas las mujeres mediante la educación y la formación en cuestiones de gobierno, políticas públicas, economía, tecnologías de la información y las comunicaciones y ciencias a fin de que desarrollen los conocimientos y las aptitudes necesarios para contribuir plenamente en todas las esferas de la vida pública; e) Proteger el derecho de las mujeres al trabajo decente combatiendo la arraigada segregación horizontal de los mercados de trabajo que favorece a los hombres y los coloca predominantemente en sectores ocupacionales con mayor reconocimiento profesional sobre la base del patrocinio más que del mérito; f) Mejorar y ampliar el acceso de las mujeres a las tecnologías de la información y las comunicaciones, incluidos los instrumentos de gobierno electrónico, a fin de posibilitar su participación política y, en general, promover su inclusión en los procesos democráticos, mejorando también la capacidad de esas tecnologías para atender las necesidades de las mujeres, en particular de las mujeres marginadas; g) Desarrollar herramientas, aptitudes y programas de formación adecuados, en consulta con las mujeres, a fin de prepararlas y empoderarlas para ocupar puestos directivos y asumir responsabilidades en la vida pública; h) Adoptar todas las medidas necesarias para eliminar los prejuicios y estereotipos de género que obstaculizan el acceso de las mujeres a las esferas social, económica y política y su plena participación en ellas. 
Objetivos de la UNESCO y la Unión Europea sobre Gender Mainstreaming. Aplicación en la docencia del Derecho mercantil

\section{Conclusión}

Comparto con la doctrina mercantilista española la inquietud por la perpetuación de los estereotipos de género en vocaciones, elección de carreras universitarias, salarios, acceso a puestos de poder, dirección y administración, barreras y techos de cristal y cemento, en los ámbitos público y privado, de forma transversal en toda la sociedad, aunque sea perceptible una -muy lenta pero constante- mejoría estadística en nuestro sistema económico y en la realidad social empresarial. Por mi parte quiero insistir en el aspecto metodológico-docente de la materia de empresa y jurídico-mercantil. La perspectiva de gender mainstreaming o de género no es seguida, realmente, ni en la metodología y ni la docencia universitarias. Dada la igualdad de géneros formal y absoluta que plantean nuestra Constitución, la Ley Orgánica de Igualdad y las restantes normas internacionales y nacionales vinculantes para el Estado español que proclaman la igualdad legal de las personas, se pasa muy por alto dicha perspectiva en los programas y libros sobre la materia mercantil y empresarial. Sin embargo, la tozuda realidad muestra que aquella pretendida igualdad formal no se traduce realmente en la práctica.

Entiendo que ha de insistirse en que todos los organismos y entidades públicas españoles resultan obligados a respetar los criterios de igualdad sustancial perseguidos por las normas imperativas nacionales e internacionales, lo que debe traducirse en una proyección normativa y práctica real de dichos criterios en el ámbito de la empresa. Considero muy importante dar a conocer entre los docentes y el resto de la comunidad investigadora la necesidad de dar cumplimiento a la exigente normativa internacional sobre los mecanismos y propuestas para evitar en lo posible la pervivencia de estereotipos y limitaciones de género que impiden, en la realidad social y económica, el acceso de todas las personas al mundo de la empresa -en todas sus facetas- en un plano de igualdad; y ello no solo por una cuestión económica o de desarrollo social que deban propiciar las Universidades ${ }^{14}$, sino, sobre todo, por una cuestión de dignidad de todas las personas. En el mismo sentido, me parece esencial modificar la estrategia metodológica y didáctica de modo que se ofrezca una mayor perspectiva de la cuestión de género en manuales y textos docentes de la materia empresarial y mercantil. Por otro lado considero que las tareas investigadoras en cuestión de género deben verse potenciadas por los Estados -en nuestro caso, el Estado español-, con el fin de incidir en los aspectos de la empresa más necesitados de corrección de las desigualdades reales del mundo práctico y para realizar propuestas críticas de medidas legales concretas para resolverlas.

14 No se trata, en mi opinion, de que las Universidades se conviertan en máquinas motoras de la economía, aunque puedan coadyuvar a los objetivos sociales y económicos del Estado. Vid. Parker, Andrew; Neary, Michael \& Lambert, Cath (2005). "Reino Unido: La educación superior en la Edad Moderna", Calidad en la Educación, 319334. 


\section{Referencias}

Gálvez Muñoz, Luis (2011). "Sipnosis artículo 14", http://www.congreso.es/consti/constitucion/indice/sinopsis/sinopsis.jsp?art=14\&tipo $=2$

Parker, Andrew; Neary, Michael \& Lambert, Cath (2005). "Reino Unido: La educación superior en la Edad Moderna", Calidad en la Educación, 319-334.

Rodríguez Coarasa, Cristina (2003). "Sipnnosis artículo 9 de la Constitución Española", http://www.congreso.es/consti/constitucion/indice/sinopsis/sinopsis.jsp?art=9\&tipo $=2$

Ruiz Franco, Rosario (2003). Mujeres y hombres en la España franquista: sociedad, economía, política, cultura, Editorial Complutense, Madrid, 118.

Telo Núñez, María (1998). “Las investigadoras de la mujer”, Diario ABC, 18 de enero de 1998.

UNESCO (2018), Informe de segumiento de la educación en el mundo, http://www.right-toeducation.org/es/news/presentaci-n-del-resumen-sobre-g-nero-2018-de-la-unesco, y eAtlas of Literacy, Unesco Institute for Statistics, https:/tellmaps.com/uis/literacy/\#!/tellmap/-601865091 